\title{
ALGEBRAIC $K$-THEORY OF HYPERBOLIC MANIFOLDS
}

\author{
BY F. T. FARRELL AND L. E. JONES ${ }^{1}$
}

\begin{abstract}
Let $\Gamma=\pi_{1} M$ where $M$ is a complete hyperbolic manifold with finite volume. We announce (among other results) that $\mathrm{Wh} \Gamma=0$ where $\mathrm{Wh} \Gamma$ is the Whitehead group of $\Gamma$. We also announce $\mathrm{Wh}_{2} \Gamma=0$, $\tilde{K}_{0}(\mathbf{Z} \Gamma)=0, K_{-n}(\mathbf{Z} \Gamma)=\mathbf{0}$ (for $n>0$ ), and $\mathrm{Wh}_{n} \Gamma \otimes \mathbf{Q}=\mathbf{0}$ (for all $n)$. We calculate the weak homotopy type of the stable topological concordance space $\mathfrak{C}(M)$, and hence Waldhausen's $\mathrm{Wh}^{P L}$-theory (cf. [22]) of $M$, in terms of simpler stable concordance spaces. When $M$ is compact, the calculation is in terms of $\mathfrak{C}\left(S^{1}\right)$ where $S^{1}$ is the circle.
\end{abstract}

A connected complete Riemannian manifold $M$ is called weakly admissible if there exist positive real numbers $a<b$ such that all the sectional curvatures of $M$ are less than $-a$ and bigger than $-b$. A weakly admissible manifold is admissible if it has finite volume. In particular, all complete locally symmetric spaces having finite volume and strictly negative sectional curvatures are admissible Riemannian manifolds. These are precisely the real, complex, quaternionic and Cayley complete hyperbolic manifolds of finite volume. All complete manifolds of constant negative sectional curvature and finite volume occur among these; in fact, they are the complete real hyperbolic manifolds of finite volume. The purpose of this paper is to announce the calculation of the algebraic $K$-theory of admissible manifolds.

We start by stating that the Whitehead group $\mathrm{Wh} \pi_{1} M$ of the fundamental group of an admissible manifold $M$ vanishes. Actually, we proceed to formulate and state a bit more general result. A group $\Gamma$ is $K$-flat if $\mathrm{Wh}\left(\Gamma \oplus C^{n}\right)=0$ for all nonnegative integers $n$ where $C^{n}$ denotes the free abelian group of rank $n$. The Bass-Heller-Swan formula $[3]$ implies $\mathrm{Wh} \Gamma=0, \tilde{K}_{0}(\mathbf{Z} \Gamma)=0$ and $K_{-n}(\mathbf{Z} \Gamma)=0$ provided $\Gamma$ is $K$-flat and $n>0$.

A smooth fiber bundle $F \rightarrow E \underset{p}{\rightarrow} M$ is admissible if

(i) $M$ is admissible;

(ii) $F$ is a closed connected manifold;

(iii) for each virtually poly-Z subgroup $S$ of $\pi_{1} M, p_{\#}^{-1}(S)$ is a $K$-flat subgroup of $\pi_{1} E$.

A group $\Gamma$ is admissible if it is isomorphic to the fundamental group of the total space $E$ of an admissible fiber bundle. The main result of [9] is: Any torsion-free virtually poly-Z group is $K$-flat. Consequently, the fundamental group of an admissible Riemannian manifold is admissible. In particular, any torsion-free discrete subgroup $\Gamma$ of the Lie group $G$, where $G$ is either $O(1, n)$,

Received by the editors August 25, 1985.

1980 Mathematics Subject Classification (1985 Revision). Primary 16A27, 18F25, 22E40.

${ }^{1}$ Both authors were supported in part by the NSF. 
$U(1, n), S p(1, n)$ or $F_{4}$, is admissible provided the measure induced on $G / \Gamma$ from Haar measure on $G$ is finite.

THEOREM 1. All admissible groups are $K$-flat.

Other groups are admissible besides fundamental groups of admissible manifolds. For instance, all torsion-free virtually poly-Z groups are admissible. Also, if $\Gamma$ is an admissible group and $M$ is a compact admissible manifold, then $\Gamma \oplus \pi_{1} M$ is admissible.

COROLlaRY 1. Let $M_{1}, M_{2}, \ldots, M_{n}$ be compact admissible manifolds and $\Gamma_{i}=\pi_{1} M_{i}$. Let $\Gamma$ be any admissible group, then $\Gamma \oplus \Gamma_{1} \oplus \Gamma_{2} \oplus \cdots \oplus \Gamma_{n}$ is admissible and hence $K$-flat.

Next, we describe the weak homotopy type of Hatcher's stable topological concordance space $\mathfrak{C}(M)$ (cf. [16]) of an admissible manifold $M$. When $M$ is compact, this is done in terms of $\mathfrak{C}\left(S^{1}\right)$ where $S^{1}$ denotes the circle.

A weakly admissible manifold $M$ is almost admissible if the lengths of closed geodesics in $M$ are bounded away from 0 . Note an admissible manifold is almost admissible. Let $M$ be an almost admissible manifold and $S_{1}, S_{2}, \ldots$ be a possibly finite sequence of circles, one for each closed geodesic in $M$. (We consider two geodesic curves to be the same geodesic if they have the same image in $M$.) Let $f_{i}: S_{i} \rightarrow M$ trace the $i$ th closed geodesic once. Define $\bigoplus_{M} \mathfrak{C}\left(S^{1}\right)$ to be the direct limit as $n \rightarrow \infty$ of the finite Cartesian products

$$
\mathfrak{C}\left(S_{1}\right) \times \mathfrak{C}\left(S_{2}\right) \times \cdots \times \mathfrak{C}\left(S_{n}\right) .
$$

THEOREM 2. If $M$ is almost admissible, then $\mathfrak{C}(M)$ weakly dominates $\bigoplus_{M} \mathfrak{C}\left(S^{1}\right)$, i.e., there are continuous maps

$$
\begin{aligned}
& f: \bigoplus_{M} \mathfrak{C}\left(S^{1}\right) \rightarrow \mathfrak{C}(M), \\
& g: \mathfrak{C}(M) \rightarrow \bigoplus_{M} \mathfrak{C}\left(S^{1}\right)
\end{aligned}
$$

such that the composite $g \circ f$ is a weak homotopy equivalence. Furthermore, the restriction of $f$ to $\mathfrak{C}\left(S_{i}\right)$ is the map induced by the geodesic curve $f_{i}$.

Assuming $M$ to be admissible, Gromov [15] has constructed a smooth manifold compactification $\bar{M}$ of $M$. Let $B_{1}, B_{2}, \ldots, B_{m}$ denote the connected components of $\partial \bar{M}$; Gromov shows each $B_{i}$ is aspherical and $\pi_{1} B_{i}$ is virtually poly-Z.

THEOREM 3. If $M$ is admissible, then there is a weak homotopy equivalence

$$
\varphi: \mathfrak{C}\left(B_{1}\right) \times \mathfrak{C}\left(B_{2}\right) \times \cdots \times \mathfrak{C}\left(B_{m}\right) \times\left(\bigoplus_{M} \mathfrak{C}\left(S^{1}\right)\right) \rightarrow \mathfrak{C}(\bar{M})
$$

such that $\varphi$ restricted to $\bigoplus_{M} \mathfrak{C}\left(S^{1}\right)$ is the map $f$ posited in Theorem 2 and $\varphi$ to $\mathfrak{C}\left(B_{i}\right)$ is the map induced by the inclusion of $B_{i}$ into $\bar{M}$.

COROLLARY 2. Let $M$ be an admissible manifold; then for each nonnegative integer $n$

$$
\mathrm{Wh}_{n}\left(\pi_{1} M\right) \oplus \mathbf{Z}[1 / N]=0
$$


where $N=[(n+1) / 2]$ !; in particular,

$$
\mathrm{Wh}_{2}\left(\pi_{1} M\right)=0 \quad \text { and } \quad \mathrm{Wh}_{n}\left(\pi_{1} M\right) \otimes \mathbf{Q}=\mathbf{0}
$$

for all $n$.

This result is a consequence of Theorem 3, the Main Theorem and Lemma 2.4 of $[\mathbf{1 7}]$, and Corollary 1.3 of [19].

Corollary 2 together with Lemma 4.4 of [8], which uses Borel's calculation [4] of $K_{n}(\mathbf{Z}) \otimes \mathbf{Q}$, yields the following calculation of $K_{n}\left(\mathbf{Z} \pi_{1} M\right) \otimes \mathbf{Q}$.

COROLLARY 3. Let $M$ be an admissible manifold and $n$ be any integer; then

$$
K_{n}\left(\mathbf{Z} \pi_{1} M\right) \otimes \mathbf{Q} \cong H_{n}(M, \mathbf{Q}) \oplus\left(\bigoplus_{j=1}^{\infty} H_{(n-1)-4 j}(M, \mathbf{Q})\right)
$$

Theorem 1 is proved in [12] and Theorems 2 and 3 in [13]. In sequels to these papers, we will pursue analogues of Theorems 1,2 and 3 for wider classes of manifolds, including complete nonpositively curved locally symmetric spaces of higher rank.

Since our most complicated argument occurs in proving Theorem 1, we limit ourselves here to a discussion of the ingredients used in its proof. First, there is the dynamics of the geodesic flow $g^{t}(t \in \mathbf{R})$ on the unit sphere bundle $S M$ of a weakly admissible manifold $M$. Here we make crucial use of work of Anosov [2]. In particular, we use that the tangent bundle TSM splits as the direct sum of subbundles $\xi^{p}, \xi^{s}$ and $\xi^{u}$, each of which is left invariant by $d g^{t}$. Furthermore, $d g^{t}$ preserves the lengths of vectors in $\xi^{p}$ (where $\xi^{p}$ is the subbundle tangent to the flow lines) and $d g^{t}$ exponentially, in positive $t$, contracts the lengths of vectors in $\xi^{s}$ and expands the lengths of vectors in $\xi^{u}$.

For a second crucial ingredient, we use the relationship of metric topology to Whitehead torsion as initiated by Connell and Hollingsworth in [7]. To be more specific, we prove, in a special case, a foliated version of Ferry's metric $h$-cobordism theorem [14]. (A fibered version had previously been proven by Quinn [18] and Chapman [6].) Roughly speaking, we show the following in the case $M$ is closed and negatively curved. Given a number $\alpha>0$, there exists a number $\delta>0$ such that any $\alpha$ controlled $h$-cobordism over $S M$ which is $\delta$ controlled in the direction perpendicular to the flow direction has zero Whitehead torsion. In fact, we generalize the fibered version to this foliated context. In particular, suppose $p: E \rightarrow S M$ is a smooth fiber bundle with fiber $F$ a closed connected manifold such that $p_{\#}^{-1}(\Gamma)$ is $K$-flat for every poly-Z by finite subgroup $\Gamma$ of $\pi_{1} S M$. If $W$ is a $h$-cobordism over $E$ which is $\alpha$ controlled over $S M$ and $\delta$ controlled perpendicular to the $g^{t}$ flow direction, then $W$ has zero Whitehead torsion. We actually generalize this fibered version further in two directions - both of which are needed to prove Theorem 1.

First, suppose $S^{+} M \rightarrow M$ is a smooth subbundle of $S M \rightarrow M$ whose fiber $S_{x}^{+} M$ at each point $x \in M$ is a closed codimension-0 disc in the unit sphere $S_{x} M$ of the tangent space $T_{x} M$. Also, assume that $S^{+} M$ is an invariant subspace under the geodesic flow $g^{t}$ on $S M$. Let $E^{+}=p^{-1}\left(S^{+} M\right)$ and 
suppose $W^{+}$is a codimension-0 submanifold of $W$ such that $W^{+}$is an $h$ cobordism over $E^{+}$. If both $W$ and $W^{+}$are simultaneously $\alpha$ controlled over $S M$ and $\delta$ controlled perpendicular to the $g^{t}$ flow direction, then the inclusion map of $E^{+}$into $W^{+}$has zero Whitehead torsion.

Second, in addition to the above, we need to allow $M$ to be noncompact (in a controlled way), but $W$ and $W^{+}$to have simultaneously product structures outside a compact subset of $E$. In addition to the metric topology techniques contained in [14, 18 and 6], the proof of this result uses ideas developed for putting combinatorial structures on hyperbolic dynamical systems, cf. $[\mathbf{1}, \mathbf{2 1}$, 20, 5, 10, and 11].

The piecing together of these two ingredients is complicated, so we will not describe it here; rather we refer the reader to [12]; $\S 0$ of $[\mathbf{1 2}]$ contains a detailed outline of the proof of Theorem 1 .

\section{REFERENCES}

1. R. L. Adler and B. Weiss, Entropy, a complete metric invariant for automorphisms of the torus, Proc. Nat. Acad. Sci. U.S.A. 57 (1967), 1573-1576.

2. D. V. Anosov, Geodesic flows on closed Riemannian manifolds with negative curvature, Proc. Steklov Inst. Math. 90 (1969).

3. H. Bass, A. Heller and R. G. Swan, The Whitehead group of a polynomial extension, Inst. Hautes Études Sci. Publ. Math. 22 (1964), 61-79.

4. A. Borel, Stable real cohomology of arithmetic groups, Ann. Sci. École Norm. Sup. (4) 7 (1974), 235-272.

5. R. Bowen, Symbolic dynamics for hyperbolic flows, Amer. J. Math. 95 (1973), 429460.

6. T. A. Chapman, Homotopy conditions which detect simple homotopy equivalences, Pacific J. Math. 80 (1979), 13-46.

7. E. H. Connell and J. Hollingsworth, Geometric groups and Whitehead torsion, Trans. Amer. Math. Soc. 140 (1969), 161-181.

8. F. T. Farrell and W. C. Hsiang, On the rational homotopy groups of the diffeomorphism groups of discs, spheres and aspherical manifolds, Proc. Sympos. Pure Math., vol. 32, Amer. Math. Soc., Providence, R.I., 1978, pp. 325-337.

9. (2) 24 (1981), 308-324.

10. F. T. Farrell and L. E. Jones, Markov cell structures, Bull. Amer. Math. Soc. 83 (1977), 739-740.

11. - Markov cell structures for expanding maps in dimension two, Trans. Amer. Math. Soc. 255 (1979), 315-327.

12.

13. _ $K$ - theory and dynamics. II (in preparation).

14. S. Ferry, The homeomorphism group of a compact Hilbert cube manifold is an $A N R$, Ann. of Math. (2) 106 (1977), 101-119.

15. M. Gromov, Manifolds of negative curvature, J. Differential Geom. 13 (1978), 223-230.

16. A. E. Hatcher, Concordance spaces, higher simple homotopy theory, and applications, Proc. Sympos. Pure Math., vol. 32, Amer. Math. Soc., Providence, R.I., 1978, pp. 3-21.

17. A. J. Nicas, On the higher Whitehead groups of a Bieberbach group, Trans. Amer. Math. Soc. 287 (1985), 853-859.

18. F. Quinn, Ends of maps. I, Ann. of Math. (2) 110 (1979), 275-331.

19. _ Algebraic K-theory of poly-(finite or cyclic) groups, Bull. Amer. Math. Soc. 12 (N.S.) (1985), 221-226. 
20. M. Ratner, Markov partitions for Anosov flows on $n$-dimensional manifolds, Israel J. Math. 15 (1973), 92-114.

21. Ya. Sinai, Markov partitions and C-diffeomorophisms, Functional Anal. Appl. 2 (1968), 61-82.

22. F. Waldhausen, Algebraic K-theory of topological spaces. I, Proc. Sympos. Pure Math., vol. 32, Amer. Math. Soc., Providence, R.I., 1978, pp. 35-60.

Department of Mathematics, State University of New York at Stony BROOK, STONY BROOK, NEW YORK 11794 (Current address of L. E. Jones)

Current address: (F. T. Farrell) Department of Mathematics, Columbia University, New York, New York 10027 
